\title{
Clinical value of early laparoscopic therapy in the management of tubo-ovarian or pelvic abscess
}

\author{
XIAOFEI JIANG ${ }^{1 *}$, MINGQING SHI $^{2 *}$, MIAO SUI $^{3}$, TAO WANG $^{1}$, \\ HAIYAN YANG ${ }^{4}$, HUIFANG ZHOU ${ }^{5,6}$ and $\mathrm{KAI} \mathrm{ZHAO}^{1}$ \\ ${ }^{1}$ Department of Gynecology, Xuzhou City Hospital of Chinese Medicine, Xuzhou, Jiangsu 221003; \\ ${ }^{2}$ Department of Obstetrics and Gynecology, Lishui Hospital of Chinese Medicine, Lishui, Zhejiang 323000; \\ Departments of ${ }^{3}$ Endocrinology and ${ }^{4}$ Science and Education, Xuzhou City Hospital of Chinese Medicine, Xuzhou, \\ Jiangsu 221003; ${ }^{5}$ Department of Gynecology, Jiangsu Provincial Hospital of Chinese Medicine, Nanjing, Jiangsu 210029; \\ ${ }^{6}$ The First Clinical Medical College of Nanjing University of Chinese Medicine, Nanjing, Jiangsu 210023, P.R. China
}

Received November 12, 2018; Accepted May 17, 2019

DOI: $10.3892 /$ etm.2019.7699

\begin{abstract}
Broad-spectrum antibiotics are the conservative treatment for tubo-ovarian abscess (TOA) or pelvic abscess, but the failure rate of antibiotic therapy remains higher in patients with a larger abscess. The present study aimed to evaluate the clinical value of early laparoscopic therapy in the management of TOA or pelvic abscess. A total of 100 patients were enrolled and their medical records were retrospectively analyzed after excluding 6 patients with malignant diseases. Based on the treatment they had received, the patients were divided into a conservative treatment group $(n=41)$ and an early laparoscopic treatment group $(\mathrm{n}=53)$. In the conservative treatment group, 21 patients $(51.2 \%)$ finally received laparoscopic exploration (late laparoscopic treatment group), and 20 patients (48.8\%) achieved a success of antibiotic therapy (successful antibiotic therapy group). The cut-off value of abscess size for predicting antibiotic treatment failure was determined using receiver operating characteristic curve analysis. Multivariate logistic regression analyses were used to explore the association between the clinical variables and antibiotic therapy failure in conservative treatment group. The durations of elevated temperature $>38.0^{\circ} \mathrm{C}$ and hospitalization were significantly longer in the conservative treatment group than those in the early laparoscopic treatment
\end{abstract}

Correspondence to: Dr Huifang Zhou, Department of Gynecology, Jiangsu Provincial Hospital of Chinese Medicine, 155 Hanzhong Road, Nanjing, Jiangsu 210029, P.R. China

E-mail: zhouhuifang_123@126.com

Dr Kai Zhao, Department of Gynecology, Xuzhou City Hospital of Chinese Medicine, 169 Zhongshan South Road, Xuzhou, Jiangsu 221003, P.R. China

E-mail: zhaokai_sunny@126.com

*Contributed equally

Key words: tubo-ovarian abscess, pelvic abscess, laparoscopy, conservative treatment, antibiotic therapy group (all $\mathrm{P}<0.001$ ). The patients in the late laparoscopic treatment group had a larger abscess size than those in the successful antibiotic therapy group $(6.2 \pm 1.8 \mathrm{~cm}$ vs. $4.8 \pm 1.4 \mathrm{~cm}$, $\mathrm{P}=0.008)$. An abscess diameter of $5.5 \mathrm{~cm}$ was obtained as the cut-off of antibiotic failure, and the sensitivity and specificity were 81.0 and $85.0 \%$, respectively. An abscess diameter of $\geq 5.5$ $\mathrm{cm}$ was independently associated with antibiotic failure (odds ratio=5.724; 95\% CI: 2.025-16.182; $\mathrm{P}=0.001)$. In conclusion, early laparoscopic treatment was associated with a better clinical prognosis than conservative treatment and late laparoscopic therapy for TOA or pelvic abscess patients.

\section{Introduction}

Tubo-ovarian abscess (TOA) is a well-known life-threatening complication of pelvic inflammatory disease (PID), and occurs in $10-15 \%$ of females affected by PID (1). Every year, 70,000 females, accounting for $1 / 3$ of females hospitalized with PID, are treated for TOA in the USA (2). In TOA, abscess and tissue necrosis form in the fallopian tubes and/or ovaries, which significantly reduces the pregnancy rate (2), which has become an important clinical issue. It is generally thought that TOA is mainly caused by infection of the lower genital tract, as well as direct spread of infection from the diverticulum, appendix and intestinal tract (3). The common pathogenic bacteria of TOA include Bacteroides, E. coli and Peptococcus (4).

With the development of antibiotics, TOA and pelvic abscesses are now treated by broad-spectrum antibiotics as the first-line treatment, and the success rate of treatment is $34-88 \%(1,5)$, while the failure rate of antibiotic therapy is higher in patients with larger abscesses (6). Surgical treatment is also necessary if patients exhibit no clinical response after antibiotic therapy for at least $72 \mathrm{~h}$ or ruptured abscess. Compared with patients who underwent antibiotic therapy in combination with image-guided drainage, part of the patients who received antibiotic therapy alone required image-guided drainage or surgical treatment eventually $(5,7,8)$. There are still no standardized guidelines as to when either antibiotic therapy, drainage or surgery is the most suitable primary option for patients with TOA or pelvic abscess. The risk 
factors for antibiotic therapy failure and ideal subsequent clinical treatment strategies are worthy of further investigation. Risk factors for adverse clinical prognosis are various among previous studies; however, they generally comprise parameters including abscess diameter, initial inflammatory biomarkers, age and smoking status $(9,10)$.

In recent years, aggressive surgical treatment has been considered to be required in a proportion of patients with TOA. In particular, the development of minimally invasive techniques has greatly advanced the surgical treatment of TOA (11). However, whether patients benefit from early laparoscopic surgery remains controversial (12). The present study aimed to retrospectively analyze patients diagnosed with TOA or pelvic abscess to further explore whether early laparoscopic treatment provides a benefit.

\section{Materials and methods}

Study design and patients. The present study was a retrospective study of patients who were initially diagnosed with TOA or pelvic abscess at the Department of Gynecology, Xuzhou City Hospital of Traditional Chinese Medicine (Xuzhou, China) between April 2014 and June 2015. The inclusion criteria were as follows: i) Symptoms of infection, including fever and pelvic pain; ii) ultrasound or magnetic resonance imaging (MRI) examination revealed a pelvic mass $\geq 3 \mathrm{~cm}$; iii) cervical motion tenderness, uterine tenderness or adnexal tenderness, abdominal tenderness, and peritoneal signs, including rebound tenderness and guarding on physical examination. The clinical and imaging diagnostic standards were according to the sexually transmitted diseases treatment guidelines (13). Patients with combined malignant diseases were excluded from the present study.

Grouping. After definite diagnosis, all patients were informed of the treatment management options, including conservative antibiotic therapy, early laparoscopic treatment and late laparoscopic treatment), and they freely selected one of the therapeutic methods after consulting with the doctors. According to the guidelines (13), conservative treatment failure was defined as patients receiving conservative treatment with little or no clinical response (oral temperature $\geq 38^{\circ} \mathrm{C}$, no decrease in abscess diameter and pelvic pain) after $72 \mathrm{~h}$ of antibiotic treatment. Patients who failed to respond to the antibiotic regimen were given laparoscopic treatment and those who completed initial antibiotic treatment but with recurrence during follow-up also received laparoscopic treatment.

Treatment strategies. During laparoscopy, patients received general anesthesia and were placed in a lithotomy position. All surgical procedures were performed by two surgeons, who had $>10$ years of relevant surgical experience. Patients in the early laparoscopic treatment group received immediate surgical treatment. Patients in the conservative treatment group received levofloxacin $500 \mathrm{mg}$ intravenously (i.v.) once daily or cefuroxime $1,500 \mathrm{mg}$ i.v. twice daily plus metronidazole $500 \mathrm{mg}$ i.v. three times daily for $72 \mathrm{~h}$, as recommended by the Chinese Medical Association (14). The effects of the conservative treatment were evaluated based on symptoms, serum white blood cell (WBC) count, and abscess size (which was determined on ultrasound or MRI). Patients who responded to the antibiotic treatment continued this treatment to a total of 10-14 days. Conservative treatment failure was defined as patients who received conservative treatment with little or no clinical response (oral temperature $\geq 38^{\circ} \mathrm{C}$, no decrease in abscess diameter, and pelvic pain) after $72 \mathrm{~h}$ of antibiotic treatment (13). Patients who failed to respond to the antibiotic regimen were subjected to laparoscopic treatment and those who completed the initial antibiotic treatment but with recurrence during follow-up also received laparoscopic treatment.

Follow-up. All patients were invited for follow-up visits by gynecological examination and ultrasound examination at the 3 rd, 6th, 12th and 24th month after treatment at the outpatient clinic. Thereafter, patients were followed up by telephone every 6-12 months for two years. All follow-ups were performed by the same gynecologist. All patients were required to provide their own telephone number and the number of a contact person to be used during active follow-up, and none of the patients was lost to follow-up within 2 years.

Data collection. Data from the medical records of the patients, including age, para, aborta, number of dilation and curettage procedures, caesarean delivery, smoking status, serum C-reactive protein (CRP) levels, serum WBC count, body temperature, abscess diameter, menopause, smoking status, presence of an intrauterine device (IUD), duration of IUD placement and previous pelvic operation, were collected. The association between these variables and the clinical prognosis of patients with TOA and pelvic abscess were evaluated. The location of abscesses, different operation types, operation time, blood loss volume, duration of temperature $\geq 38^{\circ} \mathrm{C}$, length of hospitalization and isolated microorganisms were also recorded in patients who underwent laparoscopic treatment. Bacterial culture of pyogenic fluids and drug sensitivity tests were performed for all patients who received laparoscopic treatment.

Statistical analysis. The distribution of continuous data was assessed using the Kolmogorov-Smirnov test. Normally distributed continuous data were expressed as the mean \pm standard deviation and compared using the Student's t-test. Non-normally distributed data were presented as median (range) and analyzed with the Mann-Whitney U-test. Categorical data were presented as a proportion and analyzed with the chi-squared test or Fisher's exact test, as appropriate. Receiver operating characteristics (ROC) curve analysis was used to identify the cut-off value of abscess size for antibiotic treatment failure. Multivariate logistic regression model was used to explore the association between clinical variables and antibiotic therapy failure in the conservative treatment group. All statistical analyses were performed using the SPSS 19.0 software (IBM Corp.). $\mathrm{P}<0.05$ was considered to indicate statistical significance.

\section{Results}

Patient characteristics. A total of 100 patients initially diagnosed with TOA or pelvic abscess were included in the present study. Of these, six cases (including three menopausal females) were excluded due to malignant diseases. 


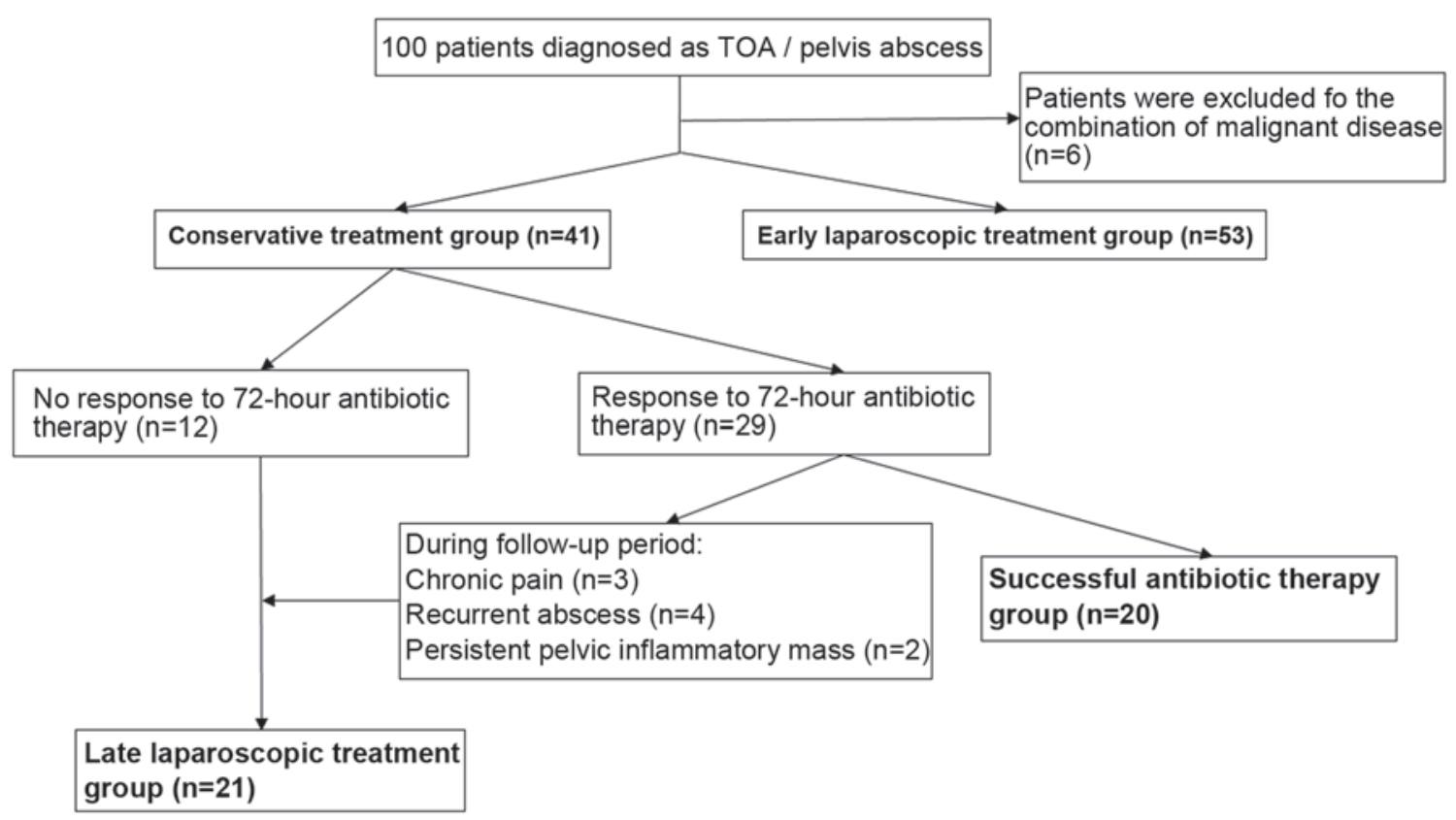

Figure 1. Patient flowchart demonstrating 53 patients were classified as early laparoscopic treatment group and 41 patients received conservative antibiotic therapy. Among the patients receiving antibiotic therapy, 20 patients achieved successful antibiotic therapy and 21 patients underwent late laparoscopic therapy. TOA, tubo-ovarian abscess.

Eventually, the data of 94 patients were analyzed. The mean age of the patients was 41 years (range, 26-60 years). A total of 53 patients opted for early laparoscopic treatment within 6-12 h of diagnosis (early laparoscopic treatment group), while 41 patients received conservative antibiotic therapy (conservative treatment group). Among the patients receiving antibiotic therapy, 29 cases were responsive to the $72 \mathrm{~h}$ conservative therapy, of which 9 patients received late laparoscopic therapy due to chronic pain $(n=3)$, recurrent abscess $(n=4)$ and persistent pelvic inflammatory mass $(\mathrm{n}=2)$. Therefore, 20 patients achieved successful antibiotic therapy (successful antibiotic therapy group). Furthermore, 12 patients failed to respond to the antibiotic therapy within $72 \mathrm{~h}$, and they received late laparoscopic therapy. Thus, a total of 21 patients underwent late laparoscopic therapy (late laparoscopic treatment group). The flow chart of the study is presented in Fig. 1. The baseline characteristics of patients in the early laparoscopic treatment group and conservative treatment group are listed in Table I. The duration of elevated temperature $>38^{\circ} \mathrm{C}$ and length of hospitalization were significantly longer in the conservative treatment group than those in the early laparoscopic treatment group (all $\mathrm{P}<0.001$ ). Regarding the other documented variables, no significant differences were observed between the two groups (all $\mathrm{P}>0.05$ ). The patients were followed up for $32.5 \pm 4.2$ and $33.8 \pm 3.4$ months in the early laparoscopic treatment group and conservative treatment group, respectively, and the difference was not statistically significant $(\mathrm{P}=0.101)$.

Comparison of the clinical characteristics between the late laparoscopic group and successful antibiotic treatment group. Of all the patients who underwent conservative antibiotic treatment, $21(51.2 \%)$ received laparoscopic exploration (late laparoscopic treatment group), including 12 cases lacking a response to antibiotics (no reduced mass size or persistent fever after $72 \mathrm{~h}$ ), two patients with persistent pelvic inflammatory mass (one in the third month and one in the fifth month, respectively), three cases with chronic pain (persisting until the fourth month in two patients and until the sixth month in one patient) and four cases with recurrent abscess (one in the third month, two in the fifth month and one in the sixth month). All of them received laparoscopic exploration between 3 days and 6 months after initiation of antibiotic treatment. The abscess size of the patients in the late laparoscopic treatment group was significantly larger than that in the successful antibiotic therapy group $(6.2 \pm 1.8 \mathrm{~cm}$ vs. $4.8 \pm 1.4 \mathrm{~cm}, \mathrm{P}=0.008)$.

Diagnosis, surgical details and vaginal culture results of the patients who received early and late laparoscopic treatment. Regarding the specific diagnosis, the patients in the early laparoscopic treatment group comprised 19 cases (35.8\%) of TOA, 26 cases (49.1\%) of pyosalpinx, 4 cases $(7.5 \%)$ of pelvic abscess, 2 cases (3.8\%) of appendicitis and 2 cases (3.8\%) of endometriosis cyst rupture complicated with infection, while the late laparoscopic treatment group included 12 cases (57.1\%) of TOA and 9 cases (42.9\%) of pyosalpinx. The types of surgeries did not demonstrate significant difference between the two groups. No organ injury was observed in either group. The results of vaginal cultures indicated that 34 patients in the early laparoscopic treatment group and 11 patients in the late laparoscopic treatment group were positive. A higher proportion of bilateral adhesion was observed in the late laparoscopic group (35.8\% vs. $61.9 \%, \mathrm{P}=0.041)$. The results are provided in Table II.

Risk factors of antibiotic failure. ROC curve analysis was performed to determine the best cut-off value of abscess diameter in order to further explore the risk of antibiotic failure. 
Table I. Comparison of patients' characteristics between early laparoscopic treatment group and conservative treatment group.

\begin{tabular}{|c|c|c|c|}
\hline Variables & $\begin{array}{c}\text { Early laparoscopic } \\
\text { treatment group }(n=53)\end{array}$ & $\begin{array}{l}\text { Conservative } \\
\text { treatment group }(n=41)\end{array}$ & P-value \\
\hline Age (years) & $39.2(25-56)$ & $38.5(24-58)$ & 0.876 \\
\hline $20-29$ & $8(15.1)$ & $6(14.6)$ & 0.823 \\
\hline $30-39$ & $20(37.7)$ & $18(43.9)$ & \\
\hline$\geq 40$ & $25(47.2)$ & $17(41.5)$ & \\
\hline Gravidity & & & 0.953 \\
\hline$\leq 2$ & $21(39.6)$ & $16(39.0)$ & \\
\hline$>2$ & $32(60.4)$ & $25(61.0)$ & \\
\hline Parity & & & 0.958 \\
\hline$\leq 1$ & $48(90.6)$ & $37(90.2)$ & \\
\hline$>1$ & $5(9.4)$ & $4(9.8)$ & \\
\hline Times of abortion & $2.1 \pm 1.5$ & $2.3 \pm 1.5$ & 0.523 \\
\hline Menopause & $2(3.8)$ & $1(2.4)$ & 0.999 \\
\hline Presence of an IUD & $27(50.9)$ & $23(56.1)$ & 0.619 \\
\hline Duration of IUD presence (years) & $7.5 \pm 5.2$ & $8.3 \pm 6.7$ & 0.529 \\
\hline Smoking & $11(20.6)$ & $7(17.1)$ & 0.999 \\
\hline Bilaterality & $19(35.8)$ & $15(36.6)$ & 0.941 \\
\hline Diameter of the abscess $(\mathrm{cm})$ & $6.0 \pm 1.7$ & $5.7 \pm 2.1$ & 0.458 \\
\hline WBC count $\left(\times 10^{9}\right.$ cells $\left./ \mathrm{mm}^{3}\right)$ & $17.3 \pm 2.8$ & $16.9 \pm 3.0$ & 0.511 \\
\hline CRP (mg/dl) & $132.5 \pm 26.8$ & $127.6 \pm 32.7$ & 0.438 \\
\hline Body temperature $\left({ }^{\circ} \mathrm{C}\right)$ & $38.6 \pm 0.7$ & $38.8 \pm 0.5$ & 0.11 \\
\hline Previous pelvic operation & $21(39.6)$ & $13(31.7)$ & 0.428 \\
\hline Appendectomy & 4 & 2 & \\
\hline Cesarean section & 11 & 8 & \\
\hline Tubal ligation & 3 & 2 & \\
\hline Ovarian cystectomy & 2 & 1 & \\
\hline Hysteromyomectomy & 1 & 0 & \\
\hline Previous pelvic inflammation history & $9(17.0)$ & $6(14.6)$ & 0.758 \\
\hline Length of hospitalization (days) & $6.8 \pm 1.5$ & $10.5 \pm 2.5$ & $<0.001$ \\
\hline Duration of body temperature $\geq 38^{\circ} \mathrm{C}$ (days) & $2.2 \pm 0.3$ & $3.2 \pm 0.8$ & $<0.001$ \\
\hline Follow-up (months) & $32.5 \pm 4.2$ & $33.8 \pm 3.4$ & 0.101 \\
\hline
\end{tabular}

Values are expressed as median (range), $\mathrm{n}(\%)$ or the mean \pm standard deviation. IUD, intrauterine device; WBC, white blood cells; CRP, C-reactive protein.

The area under the curve was 0.862 (95\% CI: 0.742-0.923), and an abscess diameter of $5.5 \mathrm{~cm}$ was obtained as the cut-off of antibiotic failure (Fig. 2). The sensitivity, specificity, positive predictive value and negative predictive value of antibiotic failure were $81,85,85$ and $81 \%$, respectively, and the Youden index was 0.66 . Multivariate regression analysis was performed to investigate the independent risk factors for failure of antibiotic therapy (Table III). Age, diameter of the abscess, serum WBC count, serum CRP, presence of an IUD, smoking status, bilaterality, menopause, caesarean delivery and previous pelvic inflammation were considered as dependent variants. The results suggested that only abscess diameter $\geq 5.5 \mathrm{~cm}$ was an independent risk factor of parenteral antibiotic therapy failure [odds ratio $(\mathrm{OR})=5.742 ; 95 \% \mathrm{CI}$ : 2.025-16.182, $\mathrm{P}=0.001]$.

\section{Discussion}

In the present study, the clinical value of early laparoscopic treatment for TOA and pelvic abscess was investigated. The results demonstrated that patients may benefit from early laparoscopic treatment. An abscess diameter of $>5.5 \mathrm{~cm}$ was a significant factor associated with failure of antibiotic treatment for pelvic abscess. The present study also suggested that patients in the early laparoscopic group achieved more rapid recovery. Compared with the patients in the conservative treatment group, the duration of body temperature $\geq 38^{\circ} \mathrm{C}$ and length of hospitalization were reduced in the early laparoscopic treatment group. Furthermore, a relatively shorter operation time and reduced blood loss volume were observed in patients of the early laparoscopic treatment group. These significant 
Table II. Diagnosis, surgical details and vaginal culture results of the patients who underwent laparoscopic surgery.

\begin{tabular}{|c|c|c|c|}
\hline Item & $\begin{array}{l}\text { Early laparoscopic } \\
\text { group }(n=53)\end{array}$ & $\begin{array}{l}\text { Late laparoscopic } \\
\text { group }(n=21)\end{array}$ & P-value \\
\hline Diagnosis & & & 0.395 \\
\hline Tubo-ovarian abscess & 19 & 12 & \\
\hline Pyosalpinx & 26 & 9 & \\
\hline Pelvic abscess & 4 & - & \\
\hline Endometriosis cyst & 2 & - & \\
\hline Appendicular abscess & 2 & - & \\
\hline Operation & & & 0.419 \\
\hline Salpingectomy & 22 & 8 & \\
\hline Simple salpingo-oophorectomy & 17 & 10 & \\
\hline Fallopian tube incision drainage & 5 & 2 & \\
\hline Pus cavity incision drainage & 5 & & \\
\hline Ovarian cystectomy & 2 & & \\
\hline Appendicectomy & 2 & & \\
\hline Hysterectomy and simple salpingo-oophorectomy & 0 & 1 & \\
\hline \multicolumn{4}{|l|}{ Positive vaginal culture } \\
\hline Escherichia coli & 24 & 7 & 0.348 \\
\hline Peptostreptococci & 8 & 4 & 0.731 \\
\hline Baumannii & 2 & 0 & 0.999 \\
\hline Bilaterality & $19(35.8)$ & $13(61.9)$ & 0.041 \\
\hline
\end{tabular}

Values are expressed $\mathrm{n}$ or $\mathrm{n}(\%)$.

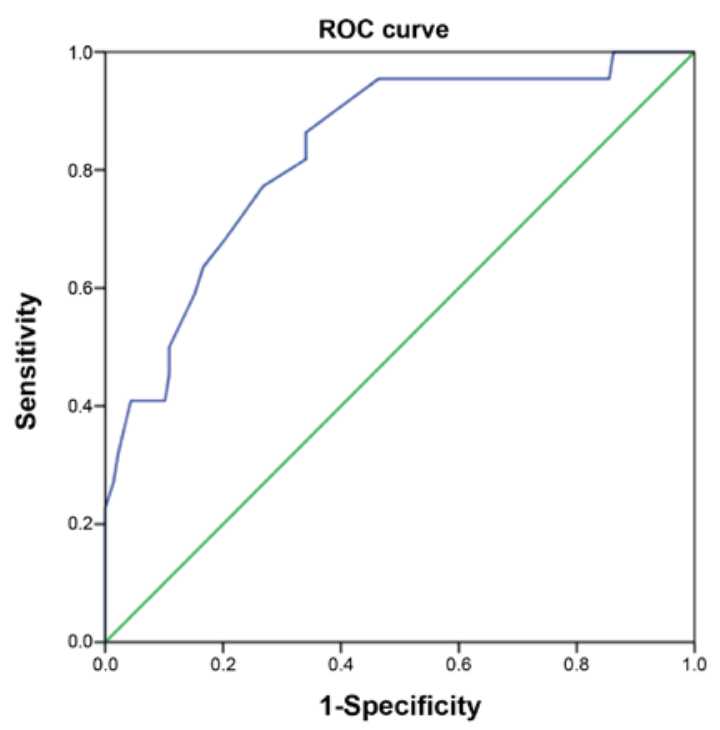

Figure 2. Cut-off value for diameter of the abscess $(\mathrm{cm})$ was calculated using ROC curve analysis. ROC, receiver operating characteristic.

differences may be associated with less severe adhesions in the early laparoscopic treatment group. A previous study indicated that antibiotic therapy resulted in prolonged time of exposure of the adnexa around purulent material (15).

It is generally recognized that the TOA and pelvic abscess may be attributed to ascending bacterial infection from the lower genital tract, causing abscess formation in the fallopian tubes, uterus and peritoneal cavity (16). TOA formation may lead to severe complications, including TOA rupture, if not treated without delay. Commonly detected bacteria from abscess aspiration or cavities are E. coli, Bacteriodes fragilis, Bacteriodes species, Peptostreptococcus, Peptococcus, and aerobic Streptococcus $(17,18)$. As the first-line medical treatment for TOA and pelvic abscess treatment, i.v. broad-spectrum antibiotics covering $N$. gonorrhoeae, $C$.trachomatis and anaerobic bacteria is recommended $(13,19)$. However, in patients diagnosed with TOA and pelvic abscess, antibiotic therapy has been reported to have a failure rate of $12-76 \%(20,21)$. Surgical treatments are necessary when patients exhibit no response or persistent mass after standard antibiotic therapy for 48-72 h. A study by Topcu et al (9) suggested that $79.8 \%$ (87/109) of patients who were managed surgically or discharged after 7 days of antibiotic therapy had a poor prognosis. The results of the present study indicated that $51.2 \%(21 / 41)$ of patients in the conservative treatment group finally received late laparoscopic surgery due to the poor response to antibiotics or chronic pain, persistent pelvic inflammatory mass or abscess recurrence.

The potential benefit of early laparoscopic treatment for patients with TOA or pelvic abscess has remained controversial. Immediate laparoscopic treatment was recommended by Roberts and Dockery (22), since it achieved a response rate of $90-100 \%$ as opposed to $20-87 \%$ in patients with conservative therapy comprising broad-spectrum antibiotics. A previous study also demonstrated that, compared with surgical intervention, the recurrence rate of TOA in patients who received antibiotic therapy was higher (23). For patients with TOA and pelvic abscess, the widely-recognized risk factor for surgical treatment after antibiotic therapy failure is abscess size, which 
Table III. Multivariate logistic regression model for clinical characteristics affecting antibiotic therapy failure in conservative treatment group.

\begin{tabular}{|c|c|c|c|c|c|c|}
\hline \multirow[b]{2}{*}{ Factor } & \multicolumn{3}{|c|}{ Univariate regression analysis } & \multicolumn{3}{|c|}{ Multivariate regression analysis } \\
\hline & OR & $95 \% \mathrm{CI}$ & P-value & OR & $95 \% \mathrm{CI}$ & P-value \\
\hline Age (years) & 1.326 & $0.830-2.119$ & 0.238 & 1.215 & $0.785-1.880$ & 0.382 \\
\hline Diameter of the abscess $\geq 5.5 \mathrm{~cm}$ & 4.274 & $1.813-10.075$ & $<0.001$ & 5.724 & $2.025-16.182$ & 0.001 \\
\hline WBC count $\left(\times 10^{9}\right.$ cells $\left./ \mathrm{mm}^{3}\right)$ & 1.534 & $0.546-4.311$ & 0.417 & 1.581 & $0.658-3.800$ & 0.306 \\
\hline $\mathrm{CRP}>50 \mathrm{mg} / \mathrm{dl}$ & 0.914 & $0.556-1.503$ & 0.723 & 0.875 & $0.502-1.526$ & 0.638 \\
\hline Presence of intrauterine device & 1.725 & $0.667-4.464$ & 0.261 & 1.291 & $0.763-2.184$ & 0.341 \\
\hline Smoking status & 1.391 & $0.632-3.060$ & 0.412 & 1.615 & $0.555-4.699$ & 0.379 \\
\hline Caesarean delivery & 0.968 & $0.877-1.069$ & 0.520 & 0.768 & $0.361-1.633$ & 0.493 \\
\hline Previous pelvic inflammation & 1.530 & $0.777-3.014$ & 0.219 & 1.135 & $0.905-1.423$ & 0.273 \\
\hline Bilaterality & 1.467 & $0.982-2.191$ & 0.061 & 2.026 & $0.921-4.455$ & 0.079 \\
\hline Menopause & 1.216 & $0.427-3.461$ & 0.714 & 1.187 & $0.535-2.632$ & 0.673 \\
\hline
\end{tabular}

OR, odds ratio; WBC, white blood cells; CRP, C-reactive protein.

may be considered an objective predictive parameter (9,24-26). In these studies, only an abscess diameter of $\geq 5-6.5 \mathrm{~cm}$ (lower limit) was considered a significant risk factor for poor response to antibiotic therapy. This may be due to the limited penetration of antibiotics into the abscess cavity $(14,27)$. The present study identified an abscess diameter of $\geq 5.5 \mathrm{~cm}$ as the independent risk factor of parenteral antibiotic therapy failure $(\mathrm{OR}=5.742$; 95\%CI: $2.025-16.182, \mathrm{P}=0.001)$, with $81 \%$ sensitivity and $85 \%$ specificity.

Menopause is a surgical indication, as in post-menopausal females, TOA and pelvic abscess may accompany a malignant tumor (19,28). Protopapas et al (29) reported a malignancy rate of $8 / 17(47 \%)$ in post-menopausal patients with TOA and $1 / 76(1.3 \%)$ in pre-menopausal patients. In the population of the present study, 7 post-menopausal patients were diagnosed with pelvic abscess, 3 of which (42.9\%) were combined with malignant tumor. However, other studies reported inconsistent results, including a Norwegian study that observed no elevated malignancy risk in post-menopausal patients and only 1 case among 16 post-menopausal patients was diagnosed with pelvic malignancy (30).

In patients who desire to maintain fertility, early laparoscopic treatment should also be considered. An extensive review of the published literature and MEDLINE was performed to investigate reasonable management options for TOA patients. Rates of responders, pregnancies and complications associated with various management strategies were compared (2). For patients with no suspected rupture, the pregnancy rates were $4-15 \%$ when treated with antibiotic therapy alone; however, the pregnancy rate reached 32-63\% if treated with antibiotics in combination with laparoscopic drainage within $24 \mathrm{~h}$. The reason for this higher pregnancy rate in patients treated by antibiotics in combination with immediate laparoscopic drainage may be associated with less adhesion around uterine adnexa, and this was also verified in a population of patients who underwent second-look laparoscopy (2). Unfortunately, the number of available studies on pregnancy outcomes after different treatments for TOA and pelvic abscess is small. While the pregnancy rate was not assessed in the present study, it indicated that more cases of bilateral adhesion were present in the late laparoscopic group.

Previous studies have investigated the risk factors of TOA and its poor outcomes, and the potential factors associated with high risk of failure of conservative treatment included age $(10,31,32)$, parity $(4,24,31)$, high levels of serum inflammatory biomarkers, e.g. WBC and $\operatorname{CRP}(8,10,25,31)$ and smoking status (10). On the contrary, the results of other studies indicated that age, levels of WBC and CRP, gravida, parity, body temperature and smoking status were not associated with antibiotic therapy failure $(9,27)$. The present study also demonstrated that there were no obvious differences in age, serum inflammatory biomarker levels, body temperature, presence of IUD, smoking status, caesarean delivery and previous pelvic inflammation between the late laparoscopic treatment group and the successful antibiotic therapy group. Certain studies reported that patients diagnosed with endometrial cyst concomitant with TOA tended to exhibit a poor response to therapeutic efficacy of antibiotics and a higher rate of late surgical treatment than those diagnosed as simple pelvic abscess $(33,34)$. However, these results were not consistent with those of another study, which suggested that endometrial cysts did not influence the antibiotic treatment (27). Thus, the risk factors influencing antibiotic therapy failure in TOA patients require further exploration.

Abscess tissues may be easily sampled for exact diagnosis through laparoscopic exploration. In the present study, the positive rate of pus bacterial culture was $60.8 \%(45 / 74)$. The commonly detected bacteria were Escherichia coli and Peptostreptococci, which was consistent with the results of a previous study (35). Appendicitis may be another differential diagnosis for patients with suspected TOA or pelvic abscess, which gynecologists should be aware of (36). In the present study, two cases of appendicitis abscess and two cases of endometriosis cyst rupture were included, and the patients received appendectomy and ovarian cystectomy, respectively. 
The surrounding tissues, including bowel, omentum majus, uterus or bladder, may adhere tightly to the abdominal wall, and these tissues may become fragile due to the presence of inflammation. However, the reported occurrence rate of bowel injuries during laparoscopic surgery is relatively low $(0.13 \%)$, which may also be repaired by laparotomy exploration (37). No bowel, bladder or urethral injury was observed in the patients of the present study. In those patients who had PID or a history of abdominal surgery, the first trocar was placed $4 \mathrm{~cm}$ above the umbilicus in order to avoid potential bowel adhesion. Furthermore, in recent years, MRI has been able to clearly display the organs adhering to the abdominal wall prior to surgery (38). In the present study, extensive adhesion of the intestinal canal prior to laparoscopic treatment was observed in four patients; the puncture point was located in the right upper quadrant and the adhesion was verified under direct vision.

The present study was not without any limitations. First, the sample size of the patients was limited and this was only a single-center study. The results require further verification in a multi-center study with a larger number of patients. Furthermore, the study design was retrospective with inherent bias, and data collection may not have been sufficient to include all relevant information, including late recurrence and late complications. Finally, the follow-up period was relatively short and the data collection and comparisons of pregnancy rate and fertility problems between conservative therapy and early laparoscopic groups were not performed and evaluated.

In conclusion, early laparoscopic treatment may be associated with better clinical prognosis than conservative treatment and late laparoscopy for patients with TOA or pelvic abscess, particularly for those with an abscess diameter of $\geq 5.5 \mathrm{~cm}$.

\section{Acknowledgements}

Not applicable.

\section{Funding}

No funding was received.

\section{Availability of data and materials}

The dataset supporting the results of the present study are included within the article.

\section{Authors' contributions}

$\mathrm{HZ}$ conceived and supervised the study; XJ, KZ and HZ performed the experiments; XJ, MS, MS and HY analyzed the data; XJ and KZ wrote the manuscript; $\mathrm{HZ}$ and $\mathrm{KZ}$ revised the manuscript. All authors reviewed the results and approved the final version of the manuscript.

\section{Ethics approval and consent to participate}

This study was approved by the clinical ethics committee of Xuzhou City Hospital of Traditional Chinese Medicine (Xuzhou, China; no. 20140305) and individual informed consent was waived for retrospective analysis.

\section{Patient consent for publication}

Not applicable.

\section{Competing interests}

All authors declare that they have no competing interests.

\section{References}

1. McNeeley SG, Hendrix SL, Mazzoni MM, Kmak DC and Ransom SB: Medically sound, cost-effective treatment for pelvic inflammatory disease and tuboovarian abscess. Am J Obstet Gynecol 178: 1272-1278, 1998.

2. Rosen M, Breitkopf D and Waud K: Tubo-ovarian abscess management options for women who desire fertility. Obstet Gynecol Surv 64: 681-689, 2009.

3. Zeger W and Holt K: Gynecologic infections. Emerg Med Clin North Am 21: 631-648, 2003.

4. Kuo CF, Tsai SY, Liu TC, Lin CC, Liu CP and Lee CM: Clinical characteristics and treatment outcomes of patients with tubo-ovarian abscess at a tertiary care hospital in Northern Taiwan. J Microbiol Immunol Infect 45: 58-64, 2012.

5. Goharkhay N, Verma U and Maggiorotto F: Comparison of CTor ultrasound-guided drainage with concomitant intravenous antibiotics vs. intravenous antibiotics alone in the management of tubo-ovarian abscesses. Ultrasound Obstet Gynecol 29: 65-69, 2007.

6. Reed SD, Landers DV and Sweet RL: Antibiotic treatment of tuboovarian abscess: Comparison of broad-spectrum beta-lactam agents versus clindamycin-containing regimens. Am J Obstet Gynecol 164: 1556-1562, 1991.

7. To J, Aldape D, Frost A, Goldberg GL, Levie M and Chudnoff S: Image-guided drainage versus antibiotic-only treatment of pelvic abscesses: Short-term and long-term outcomes. Fertil Steril 102: $1155-1159,2014$

8. Farid H, Lau TC, Karmon AE and Styer AK: Clinical characteristics associated with antibiotic treatment failure for tuboovarian abscesses. Infect Dis Obstet Gynecol 2016: 5120293, 2016.

9. Topcu HO, Kokanali K, Güzel AI, Tokmak A, Erkılınç S, Ümit $\mathrm{C}$ and Doğanay M: Risk factors for adverse clinical outcomes in patients with tubo-ovarian abscess. J Obstet Gynaecol 35: 699-702, 2015.

10. Akkurt MÖ, Yalcin SE, Akkurt I, Tatar B, Yavuz A, Yalçın Y, Akgül MA and Kayıkçıoğlu F: The evaluation of risk factors for failed response to conservative treatment in tubo-ovarian abscesses. J Turk Ger Gynecol Assoc 16: 226-230, 2015.

11. Silva F, Castro J, Godinho C, Gonçalves J, Ramalho G and Valente F: Minimally invasive approach of tubo-ovarian abscesses. Rev Bras Ginecol Obstet 37: 115-118, 2015.

12. Brun JL, Graesslin O, Fauconnier A, Verdon R, Agostini A, Bourret A, Derniaux E, Garbin O, Huchon C, Lamy C, et al: Updated French guidelines for diagnosis and management of pelvic inflammatory disease. Int J Gynaecol Obstet 134: 121-125, 2016.

13. Workowski K and Bolan G; Centers for Disease Control and Prevention: Sexually transmitted diseases treatment guidelines, 2015. MMWR Recomm Rep 64: 1-137, 2015.

14. Chu L, Ma H, Liang J, Li L, Shen A, Wang J, Li H and Tong X: Effectiveness and adverse events of early laparoscopic therapy versus conservative treatment for tubo-ovarian or pelvic abscess: A single-center retrospective cohort study. Gynecol Obstet Invest: 1-9, Jan 4, 2019 (Epub ahead of print).

15. Henry-Suchet J, Soler A and Loffredo V: Laparoscopic treatment of tuboovarian abscesses. J Reprod Med 29: 579-582, 1984.

16. Granberg S, Gjelland K and Ekerhovd E: The management of pelvic abscess. Best Pract Res Clin Obstet Gynaecol 23: 667-678, 2009.

17. Jaiyeoba O, Lazenby G and Soper D: Recommendations and rationale for the treatment of pelvic inflammatory disease. Expert Rev Anti Infect Ther 9: 61-70, 2011.

18. Wiesenfeld HC and Sweet RL: Progress in the management of tuboovarian abscesses. Clin Obstet Gynecol 36: 433-444, 1993.

19. Bevan CD, Johal BJ, Mumtaz G, Ridgway GL and Siddle NC: Clinical, laparoscopic and microbiological findings in acute salpingitis: Report on a United Kingdom cohort. Br J Obstet Gynaecol 102: 407-414, 1995. 
20. Peipert JF, Ness RB, Blume J, Soper DE, Holley R, Randall H, Sweet RL, Sondheimer SJ, Hendrix SL, Amortegui A, et al: Clinical predictors of endometritis in women with symptoms and signs of pelvic inflammatory disease. Am J Obstet Gynecol 184: 856-864, 2001.

21. Gaitán H, Angel E, Diaz R, Parada A, Sanchez L and Vargas C: Accuracy of five different diagnostic techniques in mild-to-moderate pelvic inflammatory disease. Infect Dis Obstet Gynecol 10: 171-180, 2002.

22. Roberts W and Dockery JL: Operative and conservative treatment of tubo-ovarian abscess due to pelvic inflammatory disease. South Med J 77: 860-863, 1984.

23. Dewitt J, Reining A, Allsworth JE and Peipert JF: Tuboovarian abscesses: Is size associated with duration of hospitalization \& complications? Obstet Gynecol Int 2010: 847041, 2010.

24. Kinay T, Unlubilgin E, Cirik DA, Kayikcioglu F, Akgul MA and Dolen I: The value of ultrasonographic tubo-ovarian abscess morphology in predicting whether patients will require surgical treatment. Int J Gynaecol Obstet 135: 77-81, 2016.

25. Güngördük K, Guzel E, Asicioğlu O, Yildirim G, Ataser G, Ark C, Gulova SS and Uzuncakmak C: Experience of tubo-ovarian abscess in western Turkey. Int J Gynaecol Obstet 124: 45-50, 2014.

26. Chappell CA and Wiesenfeld HC: Pathogenesis, diagnosis, and management of severe pelvic inflammatory disease and tuboovarian abscess. Clin Obstet Gynecol 55: 893-903, 2012.

27. Mizushima T, Yoshida H, Ohi Y, Ishikawa M and Hirahara F: Evaluating the risk factors for developing resistance to parenteral therapy for tubo-ovarian abscess: A case-control study. J Obstet Gynaecol Res 39: 1019-1023, 2013.

28. Workowski KA and Berman S; Centers for Disease Control and Prevention (CDC): Sexually transmitted diseases treatment guidelines, 2010. MMWR Recomm Rep 59: 1-110, 2010.

29. Protopapas AG, Diakomanolis ES, Milingos SD, Rodolakis AJ, Markaki SN, Vlachos GD, Papadopoulos DE and Michalas SP: Tubo-ovarian abscesses in postmenopausal women: Gynecological malignancy until proven otherwise? Eur J Obstet Gynecol Reprod Biol 114: 203-209, 2004.
30. Gjelland K, Ekerhovd E and Granberg S: Transvaginal ultrasound-guided aspiration for treatment of tubo-ovarian abscess: A study of 302 cases. Am J Obstet Gynecol 193: 1323-1330, 2005.

31. Greenstein Y, Shah AJ, Vragovic O, Cabral H, Soto-Wright V, Borgatta L and Kuohung W: Tuboovarian abscess. Factors associated with operative intervention after failed antibiotic therapy. J Reprod Med 58: 101-106, 2013

32. Halperin R, Levinson O, Yaron M, Bukovsky I and Schneider D: Tubo-ovarian abscess in older women: Is the woman's age a risk factor for failed response to conservative treatment? Gynecol Obstet Invest 55: 211-215, 2003.

33. Liu YT, Shi HH, Yu X, Wang S, Fan QB and Liu HY: Clinical analysis of pelvic abscess with endometriosis. Zhonghua $\mathrm{Fu}$ Chan Ke Za Zhi 52: 164-167, 2017 (In Chinese).

34. Villette C, Bourret A, Santulli P, Gayet V, Chapron C and de Ziegler D: Risks of tubo-ovarian abscess in cases of endometrioma and assisted reproductive technologies are both under- and overreported. Fertil Steril 106: 410-415, 2016.

35. Landers DV and Sweet RL: Tubo-ovarian abscess: Contemporary approach to management. Rev Infect Dis 5: 876-884, 1983.

36. Hiller N, Fux T, Finkelstein A, Mezeh H and Simanovsky N: CT differentiation between tubo-ovarian and appendiceal origin of right lower quadrant abscess: CT, clinical, and laboratory correlation. Emerg Radiol 23: 133-139, 2016.

37. van der Voort M, Heijnsdijk EA and Gouma DJ: Bowel injury as a complication of laparoscopy. Br J Surg 91: 1253-1258, 2004.

38. Ghonge NP and Ghonge SD: Computed tomography and magnetic resonance imaging in the evaluation of pelvic peritoneal adhesions: What radiologists need to know? Indian J Radiol Imaging 24: 149-155, 2014.

This work is licensed under a Creative Commons Attribution-NonCommercial-NoDerivatives 4.0 International (CC BY-NC-ND 4.0) License. 